\title{
Las chullpas incas en las quebradas Ucriamayo y Tulpaisa, Caylloma, Arequipa
}

\author{
Recibido: 23 abril $2020 \quad$ Aprobado: 23 julio 2020
}

Denis E. Correa-Trigoso

Nacionalidad: Peruano. / Université de Rennes 1

Correo: dcorreatrigoso@gmail.com. / ORCID ID: 0000-0002-1262-1432

\section{Resumen}

En las inmediaciones de la Unidad Minera (U. M.) Tambomayo se ubican las quebradas de Ucriamayo y Tulpaisa; y asociadas a estas áreas geográficas se registraron mediante la prospección arqueológicas los sitios arqueológicos Gentilmach'ay y Pushkanawasi. La zona se localiza en la parte alta del valle del Colca (área de influencia de la etnia Collagua), en el distrito de Tapay, provincia de Caylloma, departamento de Arequipa. Los sitios corresponden a estructuras chullparias con presencia de material cultural, como fragmentos de cerámica, restos vegetales, textiles, óseo animal y humano. La filiación cultural del material cerámico es inca y se observa la modificación cultural de cráneos. Ambos sitios presentan particularidades que sugiere la existencia de diferencias sociales de los individuos enterrados en los diferentes ambientes. Según las evidencias registradas, se proponen diferentes investigaciones con el material cultural registrado en ambos sitios arqueológicos.

\section{Palabras clave:}

Inca, Caylloma, Arequipa, Ucriamayo, Tulpaisa. Chullpas.

\section{The Incas Chullpas in the Ucriamayo And Tulpaisa Brokens, Caylloma, Arequipa}

\begin{abstract}
In the immediate vicinity of the Mining Unit (M. U.) Tambomayo are located the Ucriamayo and Tulpaisa streams; and associated to these geographical areas, the archeological surveys Gentilmach'ay and Pushkanawasi were registered through archeological exploration. The area is located in the upper part of the Colca Valley (area of influence of the Collagua ethnic group), in the district of Tapay, province of Caylloma, department of Arequipa. The sites correspond to chullparia structures with the presence of cultural material, such as fragments of ceramics, plant remains, textiles, animal and human bone. The cultural affiliation of the ceramic material is Inca and the cultural modification of a skulls is observed. Both sites have particularities that suggest the existence of social differences of individuals buried in different environments. According to the evidences registered, different investigations with the cultural material registered in both archaeological sites are proposed.
\end{abstract}

\section{Keywords}

Inca, Caylloma, Arequipa, Ucriamayo, Tulpaisa. Chullpas.

Datos del autor

Magister. 


\section{Introducción}

En las quebradas Ucriamayo (tributario del río Molloco) y Tulpaisa (afluente de la quebrada antes mencionada), localizados en las inmediaciones de la U. M. Tambomayo, se registraron los sitios arqueológicos de Gentilmach'ay y Pushkanawasi. Políticamente se encuentran ubicadas entre los anexos de Punachica y Tocayo, en el distrito de Tapay, provincia Caylloma, departamento de Arequipa (Figura 01). La zona geográfica corresponde a la franja medirional de la Cordillera Occidental de los Andes, con una altitud superior a los $3100 \mathrm{msnm}$ y localizada al sur del Perú. Con respecto al clima de este territorio, es húmedo-frio con variaciones entre las temporadas de friaje (julio y agosto) y de lluvias (enero y marzo). La vegetación presente en esta región está compuesta por cactáceas, gramíneas, ichu y otras hierbas de desarrollo temporal. Las actividades modernas de los pobladores se basan en la ganadería lanar (camélidos y ovinos) y aprovechan la presencia de bofedales para el pastoreo.

Figura 01: Ubicación de los sitios arqueológicos Gentilmach’ay y Pushkanawasi.

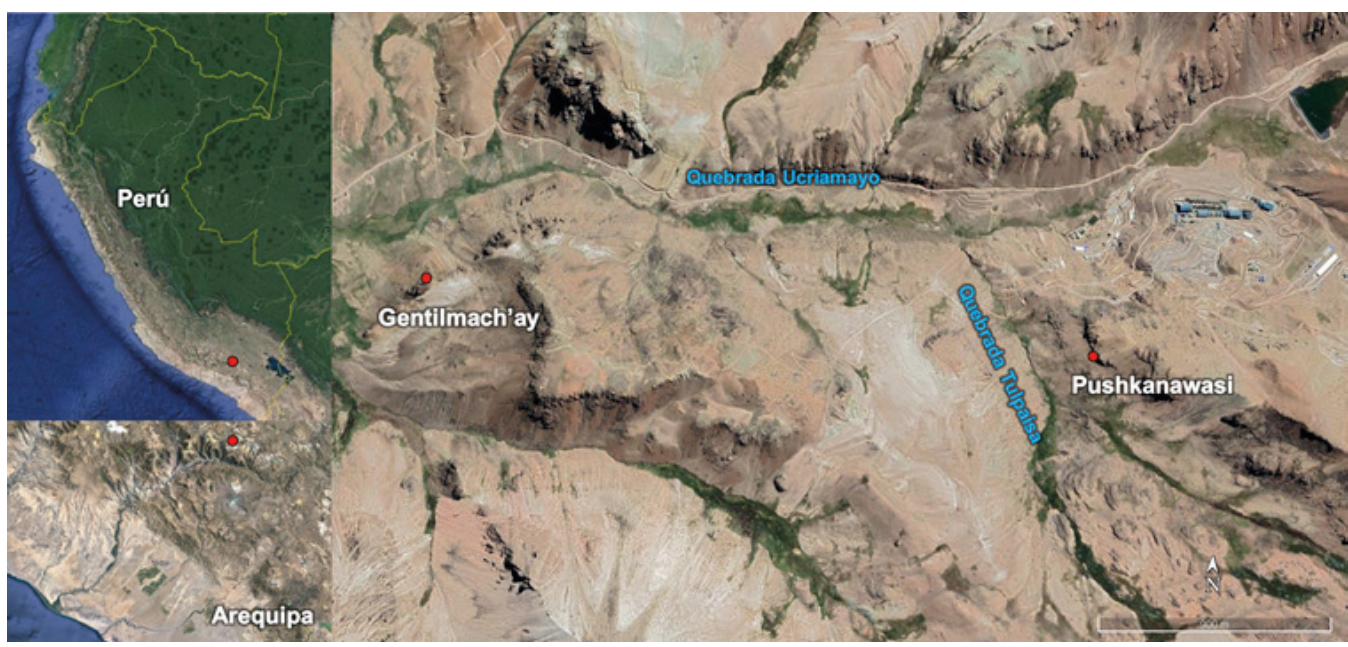

En las áreas circundantes a las quebradas Ucriamayo y Tulpaisa, se registran diversos monumentos arqueológicos, conformados por corrales, paravientos, estructuras en abrigos rocosos, chullpas, estructuras circulares y rectangulares. Asimismo, fue posible registrar material cultural mueble en la superficie, como fragmentería de cerámica, lascas y desechos líticos, restos de textiles y óseos. Según la ubicación de las estructuras y sus características arquitectónicas es posible asociar que durante el periodo prehispánico fue el pastoreo uno de los principales ejes económicos, siendo para ello aprovechados las partes altas y bajas de las quebradas (Asencios, 2015; Castilla, 2016).

Los antecedentes más resaltantes de la provincia de Caylloma durante la época pre inca están relacionadas con la coexistencia y la autosustenabilidad de dos etnias: Collaguas y Cabanas. Los primeros estaban conformados por los habitantes de las zonas medias y altas del valle de Colca, hablaban aimara y estaban especializados en la crianza de camélidos; mientras que los segundos, hablaban quechua, habitaban la zona baja del valle y eran agricultores (Doutriaux, 2002; Robles, 2008). Ambos grupos realizaban intercambios comerciales con otros pueblos y aprovecharon los beneficios que les otorgaban la variedad de pisos ecológicos 
presentes en esta región. Para el periodo Inca, según los cronistas Garcilaso de la Vega, indica que la conquista de este territorio fue mediante campañas militares realizadas durante el reinado del Inca Mayta Capac; mientras que Blas Valera, menciona que la integración se realizó mediante la alianza matrimonial entre el mismo inca con la hija del curaca de los Collaguas, Ilamada Mama Tancaray- Yacchi (Becerra et al. 2005).

\section{Metodología}

Los trabajos estuvieron asociados con la prospección arqueológica sin recolección de material en superficie en las quebradas de Ucriamayo y Tulpaisa, siendo identificado los sitios arqueológicos de Gentilmach'ay y Pushkanawasi.

\section{Resultados Gentilmach'ay}

El Sitio Arqueológico Gentilmach'ay (la cueva del gentil, en quechua) está localizado en el margen izquierdo de la quebrada Ucriamayo, en una sección del afloramiento rocoso de la ladera oeste del cerro Salhualque. Se encuentra a una altitud de 4,655 msnm y cuenta con las siguientes coordenadas UTM (WGS-84/19S): E 183879.1517 / N 8287110.9534. Presenta unas dimensiones aproximadas de $5.50 \mathrm{~m}$ de ancho por $15 \mathrm{~m}$ de largo, con una orientación de oeste a este. En el sitio se observa en superficie fragmentos de cerámica, restos óseos humanos y textiles. Se registraron ocho estructuras: cinco chullpas, una plataforma y dos ambientes circulares (Figura 02).

\section{Figura 02: Vista de oeste a este del sitio arqueológico Gentilmach'ay (A) y el registro de planta sus ambientes (B).}

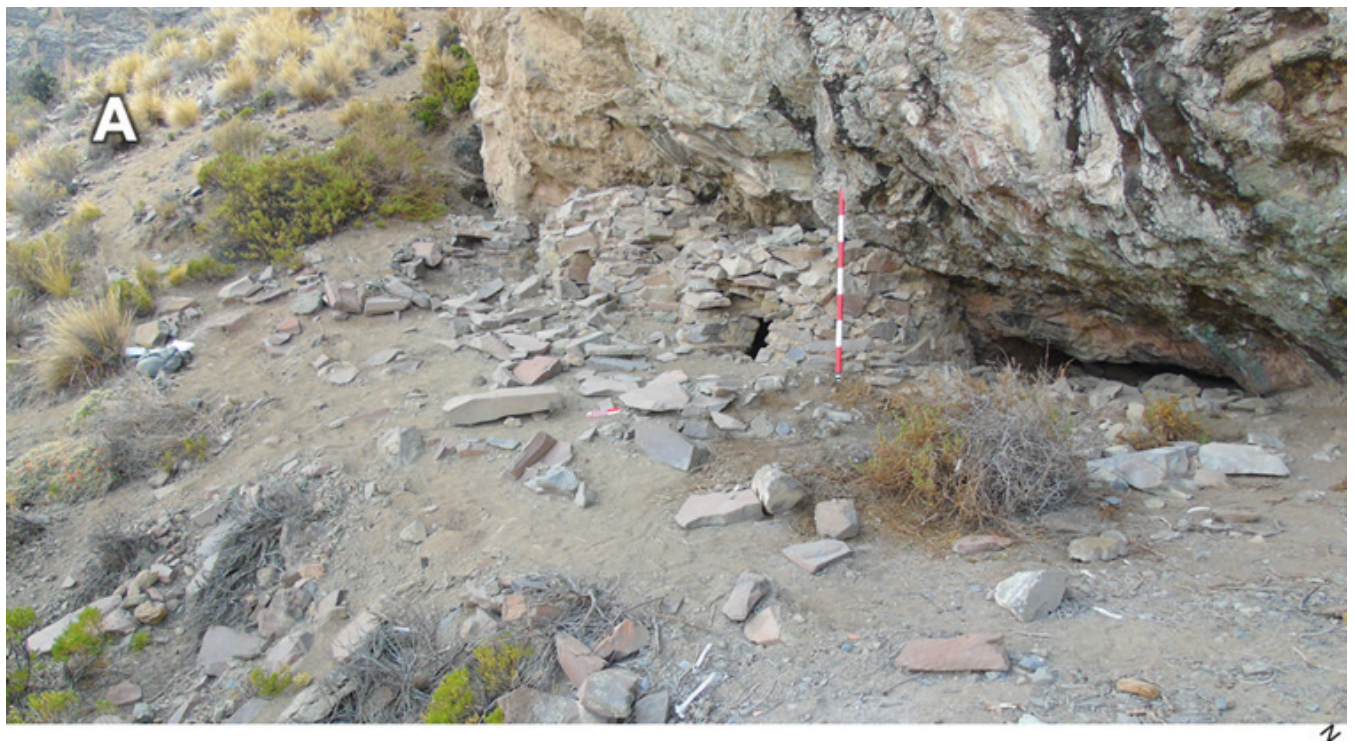




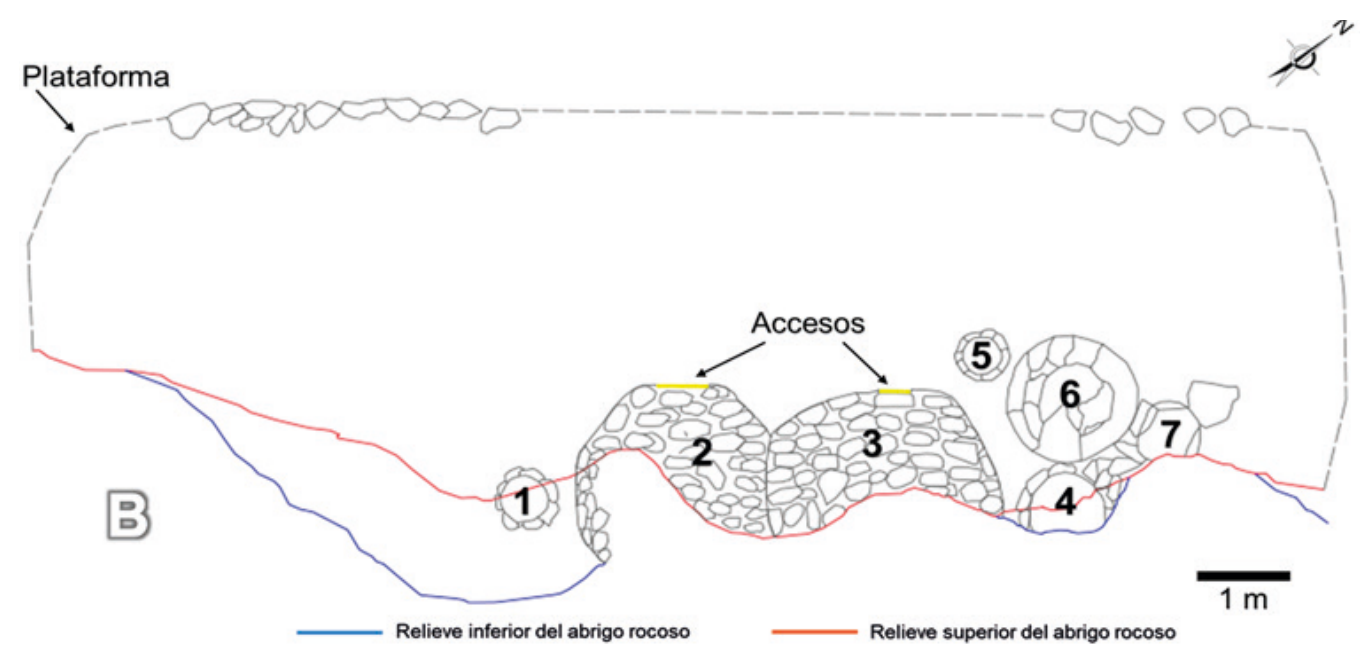

\section{Descripción arquitectónica}

Ambiente 1: Se encuentra localizada en la parte oeste del abrigo rocoso. Corresponde a una estructura circular, con un diámetro de $0.72 \mathrm{~m}$ y una profundidad conservada de $0.19 \mathrm{~m}$. Para su edificación se usaron piedras sin cantear con medidas aproximadas de $0.12 \mathrm{~m}$ de ancho por $0.08 \mathrm{~m}$ de largo y con mortero de $0.04 \mathrm{~m}$ de espesor. La estructura fue construida por debajo de la superficie. El ambiente en su totalidad fue construido y no se utilizó la saliente del abrigo rocoso para su elaboración. En su interior no se registró material cultural mueble.

Ambiente 2: Se encuentra localizada en la parte oeste del abrigo rocoso, próximo al ambiente 1. Por las características observadas en superficie, la estructura circular puede considerarse una chullpa. Presenta una altura de $1.30 \mathrm{~m}$ y un diámetro de $2.16 \mathrm{~m}$. Está edificada con piedras canteadas y sin trabajar, de medidas promedios de $0.33 \mathrm{~m}$ de largo por 0.19 de ancho. Entre las lajas de piedras presenta un mortero de $0.06 \mathrm{~m}$ de espesor. El acceso se encuentra ubicado al norte del ambiente, con un ancho de $0.62 \mathrm{~m}$ y una altura superficial de $0.48 \mathrm{~m}$ (Figura 03). Los actos de saqueo dañaron al ambiente, a tal grado que para acceder al interior de este ambiente se retiró el dintel, el cual fue posible identificar a unos metros de la estructura (Figura 04). Para su construcción se utilizó la saliente del abrigo rocoso, conformando la parte sur de la chullpa; este comportamiento es recurrente en la construcción de los ambientes 3, 4 y 7 (Figura 05). En su interior se registraron restos óseos humano, fragmentos de cerámica y textiles.

Ambiente 3: Se encuentra en la parte central del abrigo rocoso, próximo al ambiente 2 y por las evidencias registradas se consideró como una chullpa. Presenta una altura de $1.34 \mathrm{~m}$ y un diámetro de $2.60 \mathrm{~m}$. Está construida con piedras trabajadas y sin cantear, de medidas promedios de $0.30 \mathrm{~m}$ de largo por $0.12 \mathrm{~m}$ de ancho. Entre las lajas de piedras presenta un mortero de $0.04 \mathrm{~m}$ de espesor. El acceso se ubica al norte del ambiente, con un ancho de $0.39 \mathrm{~m}$ y una altura superficial de $0.51 \mathrm{~m}$. En comparación con el anterior ambiente, fue posible registrar la estructura en su totalidad y se utilizó la saliente del abrigo rocoso para delimitar la parte sur de la chullpa (Figura 03). En su interior se evidencia restos óseos humano y fragmentos de cerámica. 
Figura 03: Vista norte a sur del acceso al ambiente 2 (A) у 3 (B).

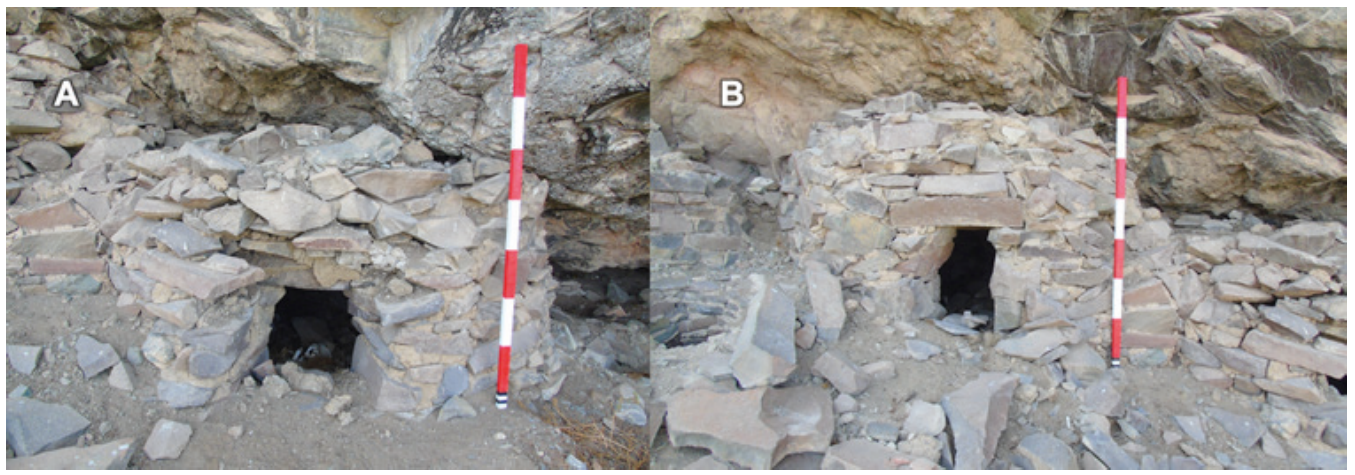

Figura 04: Ambiente 2 en el sitio Gentilmach'ay, se evidencia el retiro del dintel por parte de los saqueadores con la finalidad de facilitar el acceso.

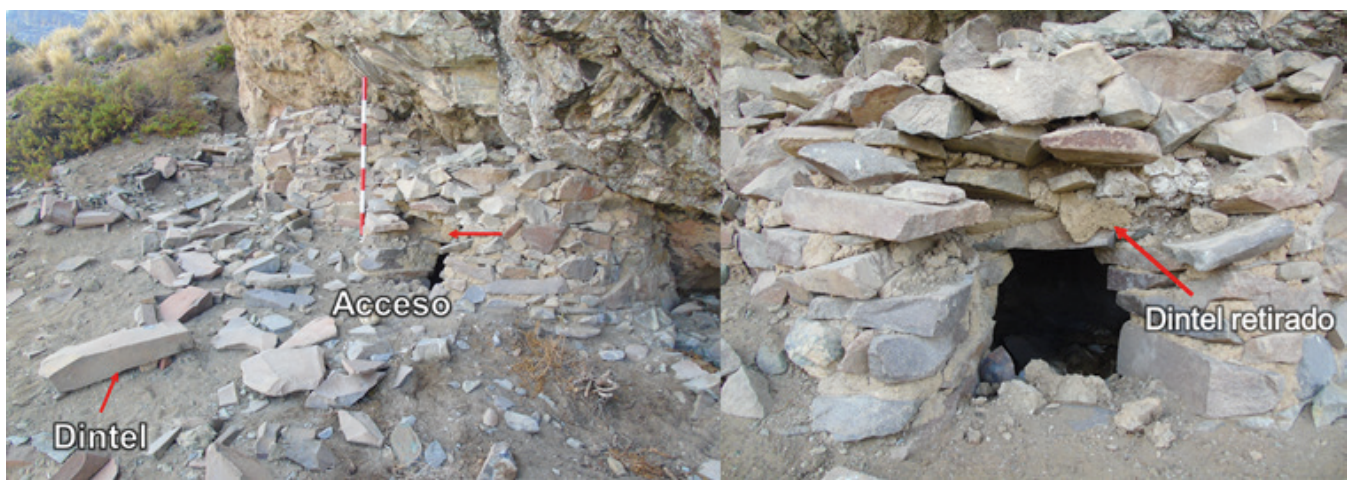

Ambiente 4: Se encuentra localizada en la parte central del abrigo rocoso y entre los ambientes 3 y 6 . Corresponde a una estructura semicircular, con una altura de $0.42 \mathrm{~m}$ y un diámetro de $0.82 \mathrm{~m}$. Por el contenido en su interior se consideró a este ambiente como una chullpa. Se encuentra construida con piedras sin cantear con medidas aproximadas de 0.31 de largo $\mathrm{m}$ por $0.11 \mathrm{~m}$ de ancho y con mortero de $0.04 \mathrm{~m}$ de espesor. Aunque en la actualidad este ambiente se encuentre disturbado y expuesto, fue posible determinar que la estructura en un principio fue de mayor altura y el sellado fue adosando la estructura a la saliente del abrigo rocoso. Prueba de ello se registra en la misma superficie de la roca, donde se puede registrar el mortero que fue empleado para su elaboración (Figura o6). El ambiente utilizó la saliente del abrigo rocoso para delimitar la parte sur. En su interior se registraron restos óseos humano y fragmentos de cerámica. 
Figura 05: Vistas en el interior de los ambientes 2 (izquierda) y 3 (derecha), donde se observa la adaptación del abrigo rocoso para la construcción de las estructuras.

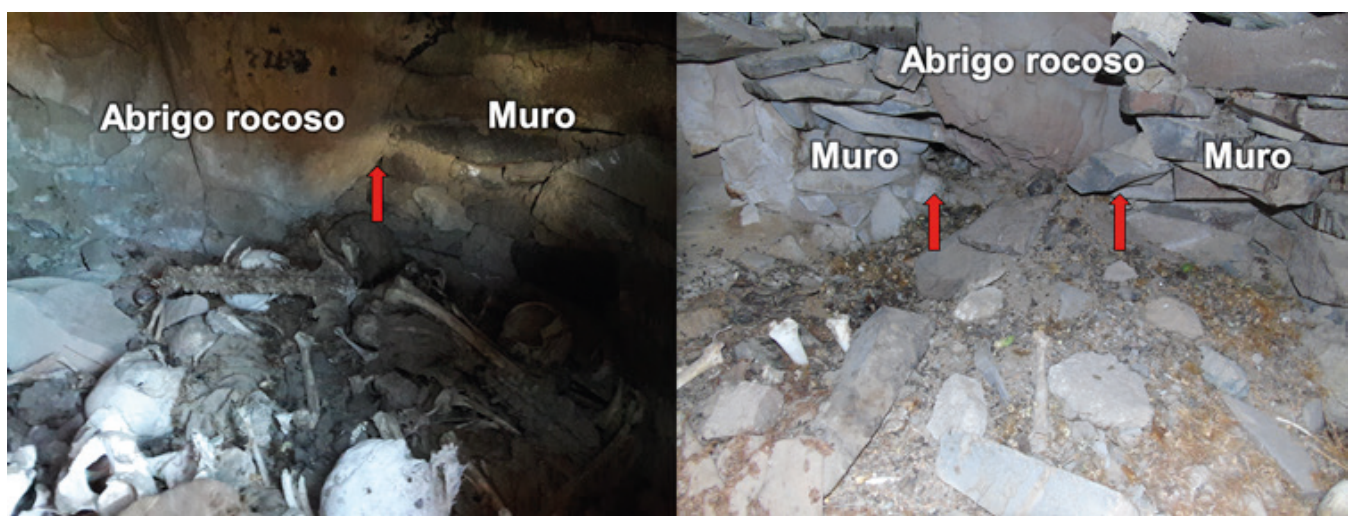

Figura 06: Restos del mortero utilizado en la construcción de la estructura 4.

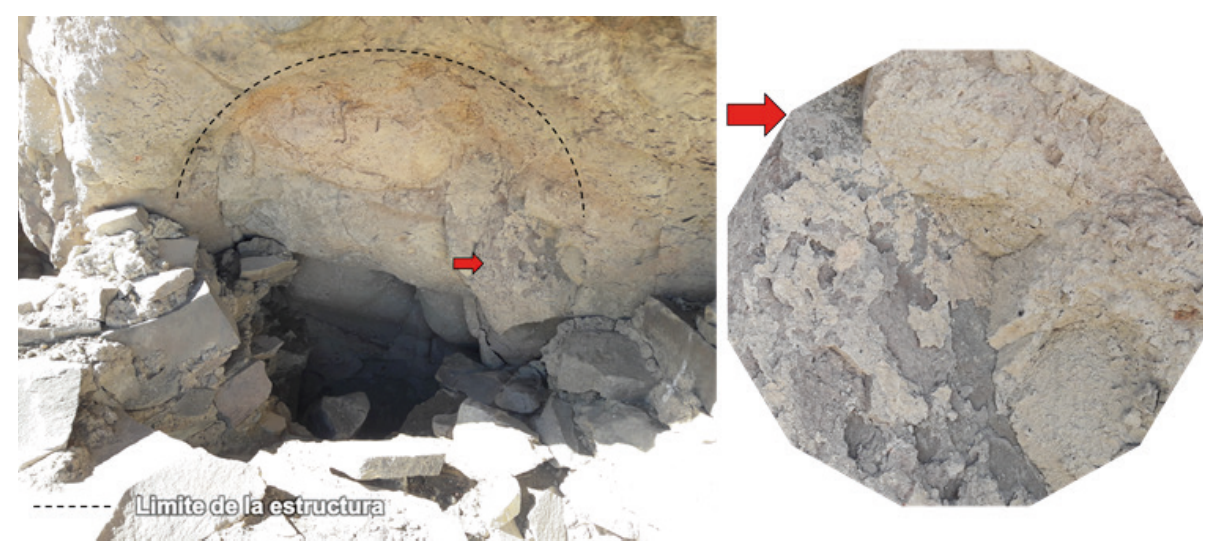

Ambiente 5: Se encuentra localizada en la parte central del abrigo rocoso y entre los ambientes 3 y 6 . Corresponde a una estructura circular, con un diámetro de $0.45 \mathrm{~m}$ y una profundidad conservada de $0.25 \mathrm{~m}$. Se construyó con piedras sin cantear con medidas aproximadas de $0.21 \mathrm{~m}$ de largo por $0.11 \mathrm{~m}$ de ancho y con mortero de $0.03 \mathrm{~m}$ de espesor. La estructura fue edificada por debajo de la superficie y no es posible definir si se encontraba sellada, al igual que el ambiente 1, los actos de saqueo destruyeron la parte superior de la estructura. Para su construcción no se utilizó ninguna saliente del abrigo rocoso. En su interior no se registró material cultural mueble.

Ambiente 6: Se encuentra localizada en la parte este del abrigo rocoso, entre los ambientes 4 y 7. También se consideró a este ambiente como una chullpa, ya que cuenta con características semejantes con los ambientes 2 y 3 . Presenta una altura de $0.64 \mathrm{~m}$ y un diámetro de 1.58 $\mathrm{m}$. Para su construcción se utilizaron piedras sin cantear con medidas promedios de $0.32 \mathrm{~m}$ de largo por 0.15 de ancho. Entre las lajas de piedras presenta un mortero de $0.06 \mathrm{~m}$ de espesor. No se logró reconocer el acceso, ya que la parte norte se encuentra cubierta por tierra. Para su edificación no se utilizó ninguna saliente del abrigo rocoso. En su interior no se registraron evidencias de material cultural mueble. 
Ambiente 7: Se encuentra localizada en la parte este del abrigo rocoso y próximo al ambiente 6. Corresponde a una estructura semicircular, con una altura de $0.40 \mathrm{~m}$ y un diámetro de $0.65 \mathrm{~m}$. Por el contenido en su interior se consideró a este ambiente como una chullpa, aunque se encuentre parcialmente destruida. Para su construcción se utilizaron piedras sin cantear con medidas aproximadas de $0.35 \mathrm{~m}$ de largo por $0.14 \mathrm{~m}$ de ancho y con mortero de $0.04 \mathrm{~m}$ de espesor. A pesar del saqueo, se observa la mitad del techo y parte del ambiente se encuentra sepultado por deslizamiento de tierra. El ambiente utilizó la saliente del abrigo rocoso para delimitar la parte sur. En su interior se registraron restos óseos humano.

Plataforma: Se encuentra ubicada en toda la dimensión del abrigo rocoso y extendiéndose hacia el norte. Presenta una orientación de oeste a este, con un largo de $15.18 \mathrm{~m}$, un ancho de $5.00 \mathrm{~m}$ y una altura aproximada de $0.40 \mathrm{~m}$. En la cara norte se utilizaron piedras como límite de la estructura, estas fueron sin cantear y no se utilizó mortero. Sobre esta plataforma se construyeron los ambientes antes descritos.

\section{Materiales en superficie}

En superficie fue posible reconocer tres tipos de materiales culturales muebles: cerámica, textil y óseo. Los fragmentos de bordes, cuerpos decorados, bases corresponden a platos, ollas con asa cintada, cuencos y vasos. Con respecto a la decoración se observa la utilización de los colores blanco-crema, negro y rojo inglés; siendo plasmados en cinco tipos: figuras zoomorfas, fitomorfas, triángulos, círculos y líneas (Figura 07). Según los fragmentos, corresponden a cerámica fina y doméstica, con coloración naranja (cocción oxidante) y gris (cocción reductora). El estilo identificado en ambos sitios arqueológicos pertenece al Inca (Fernández 1980, ML 2010), en asociación con el Horizonte Tardío (1476 - 1532 d. C). De igual forma, se registraron fragmentos de textiles de color marrón claro, elaborados con fibra animal con la técnica de faz de trama (la densidad de los hilos de trama es mayor a la densidad de los hilos de urdimbre) y torsión en "S", hacia la derecha (Figura 08).

Figura 07: Fragmentos de cerámica registrados en el sitio Gentilmach'ay y es posible asociar sus colores y la forma de los diseños con el estilo Inca. La escala corresponde a $\mathbf{2} \mathbf{c m}$.

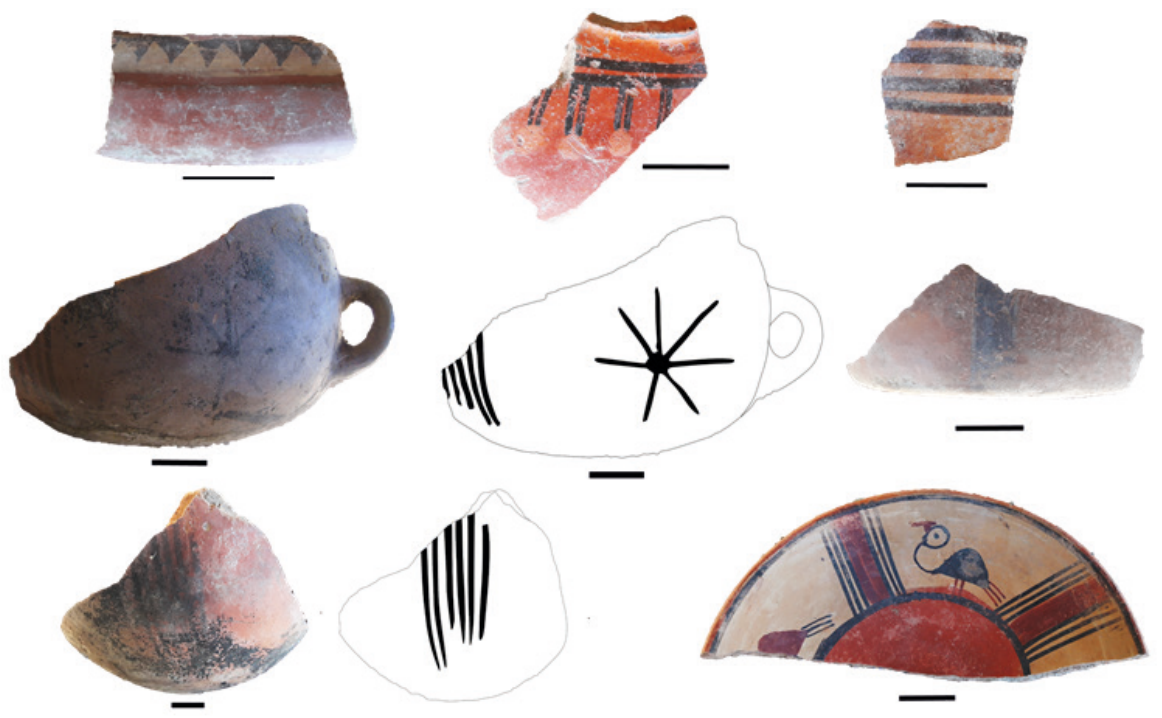


Fue posible identificar dentro de los ambientes restos óseos humanos, con mayor abundancia en los ambientes 2 y 3 (Figura o9). En el ambiente 2, mediante el conteo de cráneos, se registra un mínimo de 15 individuos y según la forma de algunos cráneos es posible identificar que presenta modificaciones culturales. Por la coloración blanquecina de los restos óseos se determina que estuvieron expuestos por mucho tiempo. También se logró observar huesos de color crema, por lo tanto, indicaría que actualmente continúan los saqueos. Similares características son registradas en el ambiente 3, con la diferencia que solo se logró determinar una cantidad mínima de cinco individuos.

Figura 08: Restos de textil registrado en el sitio arqueológico Gentilmach'ay. A: Decoración lineal con otra tonalidad en el extremo del textil y B: Tramado en del textil registrado elaborado con fibra animal (camélido).
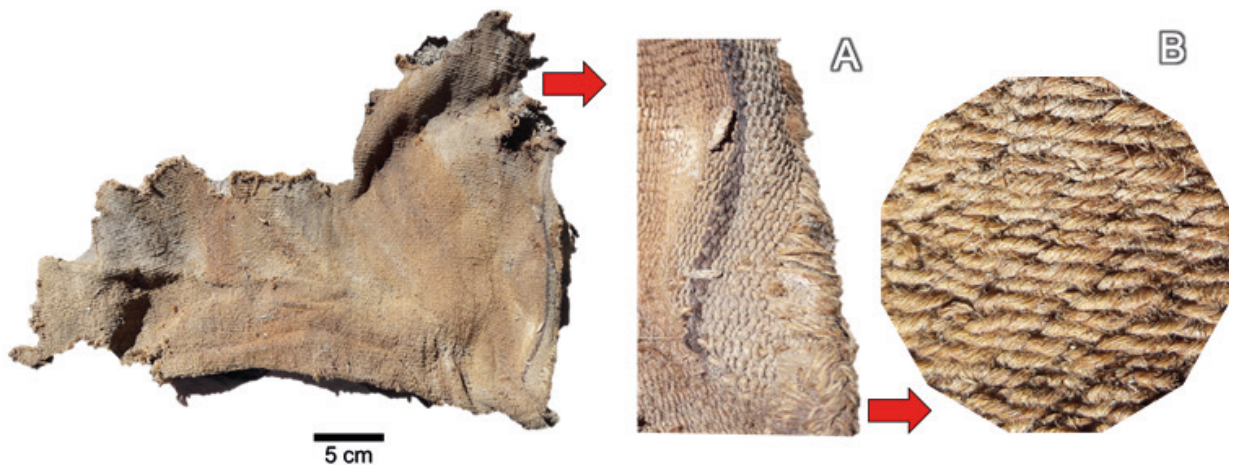

Figura 09: Restos óseos humano en el interior de los ambientes 2 (A) y 3 (B). La flecha indica un cráneo con deformación.

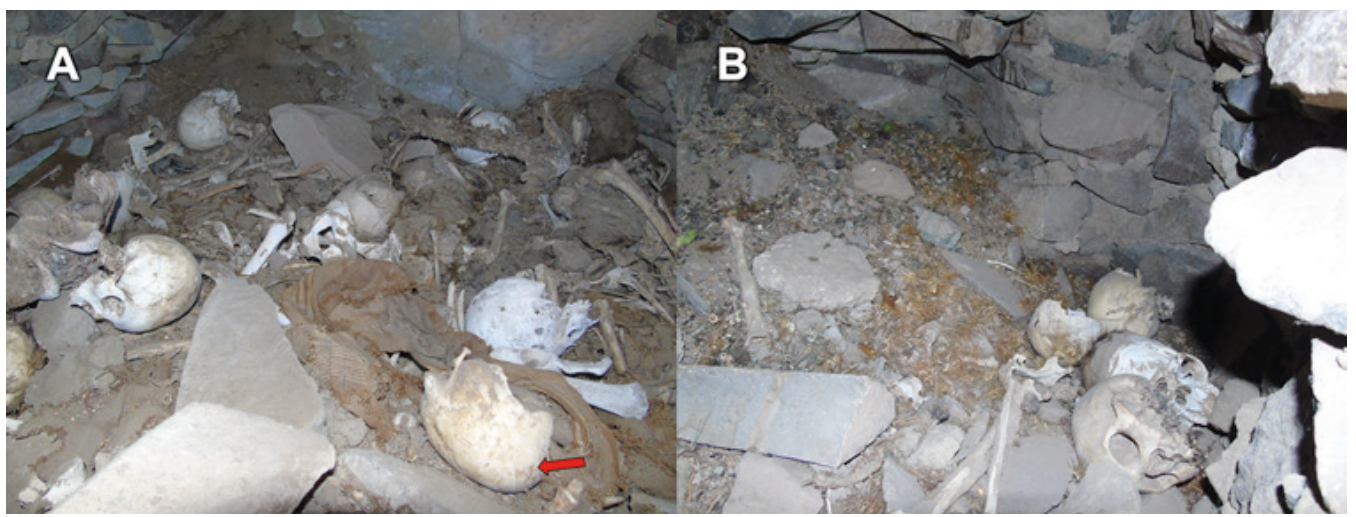

\section{Pushkanawasi}

El sitio arqueológico Pushkanawasi (la casa de la hilandera, en quechua) está localizado en el margen derecho de la quebrada Tulpaisa, en la ladera oeste del cerro adyacente a la quebrada. Se encuentra a una altitud de 4,907 msnm y cuenta con las siguientes coordenadas UTM (WGS-84/19S): E 186944.552 / N 8286909.442. Presenta unas dimensiones aproximadas de $4 \mathrm{~m}$ de ancho por $10 \mathrm{~m}$ de largo, con una orientación de norte a sur. En superficie es posible observar fragmentos de cerámica, restos óseos y vegetales. El sitio arqueológico se conforma por dos chullpas (Figuras 10). 
Figura 10: Vista oeste -este del sitio arqueológico Pushkanawasi.

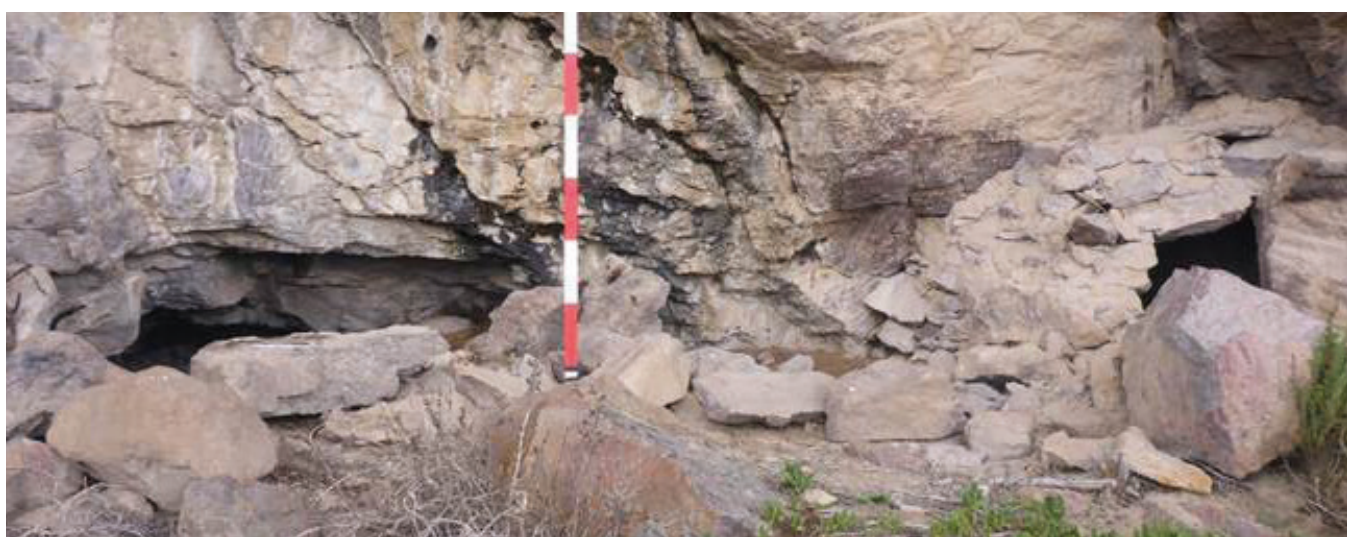

\section{Descripción arquitectónica}

Ambiente 1: Se encuentra localizada en la parte sur del sitio arqueológico. Corresponde a una estructura semicircular, con un diámetro de $1.45 \mathrm{~m}$ y una altura conservada de $0.93 \mathrm{~m}$. Se encuentra construida con piedras sin cantear con medidas aproximadas de $0.08 \mathrm{~m}$ de ancho por $0.15 \mathrm{~m}$ de largo y con mortero promedio de $0.04 \mathrm{~m}$ de espesor. El acceso se ubica al oeste del ambiente con un ancho de $0.43 \mathrm{~m}$ y una altura superficial de $0.70 \mathrm{~m}$. La estructura fue edificada usando como límite este la pared rocosa del afloramiento y en su interior no se registró material cultural mueble (Figura 11).

Ambiente 2: Se encuentra localizada en la parte norte del sitio arqueológico. Corresponde a una estructura semicircular, con un diámetro de $0.72 \mathrm{~m}$ y una altura conservada al interior de $0.19 \mathrm{~m}$. Se encuentra edificada mediante el uso del abrigo rocoso, delimitando el acceso del ambiente con piedras sin cantear con medidas aproximadas de $0.20 \mathrm{~m}$ de ancho por $0.10 \mathrm{~m}$ de largo y sin mortero. El acceso se ubica al oeste del ambiente con un ancho de $1.05 \mathrm{~m}$ y una altura superficial de $0.40 \mathrm{~m}$, estando desmontado por las actividades de saqueo (Figura 11). Al interior de la estructura se registró material cultural mueble como fragmentos de cerámica, restos óseos y objetos de madera.

Figura 11: Vista oeste -este del ambiente 1 (A) y 2 (B) en el sitio arqueológico Pushkanawasi.

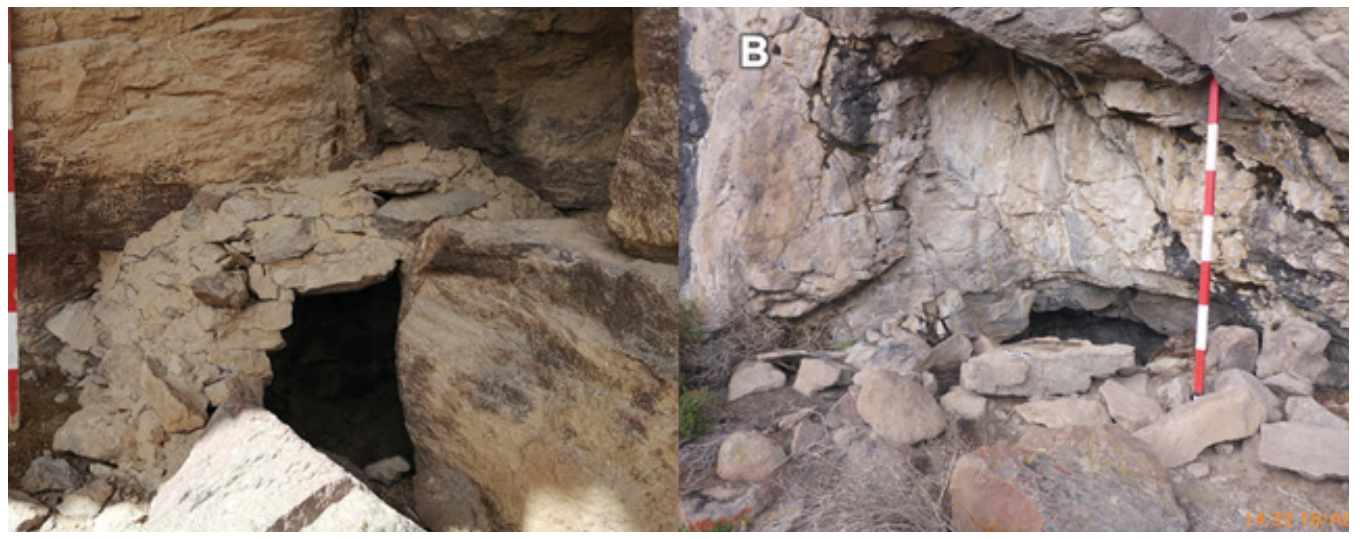




\section{Figura 12: Fragmento de cerámica (A) y una rueca de madera registrado en el ambiente 2,} en el sitio arqueológico Pushkanawasi.
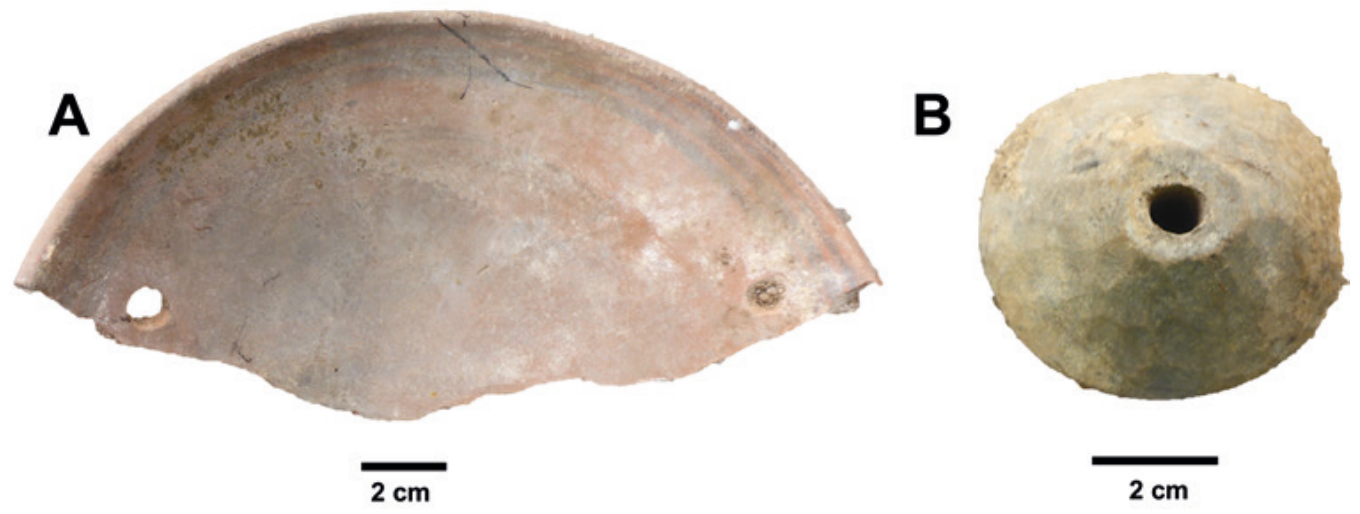

\section{Materiales en superficie}

En superficie se reconoció tres tipos de material cultural mueble: cerámica, vegetal y óseo. La cerámica identificada corresponde a fragmentos de platos y material no diagnóstico. Con respecto a la decoración se registra la presencia de líneas de color negro con una pasta de coloración naranja (cocción oxidante). Al igual que el sitio Gentilmach'ay, el motivo representado se asocia con el estilo Inca. Además, se registra un peso de hilar o rueca de madera, siendo un objeto posiblemente asociado con el ajuar funerario de los individuos enterrados dentro del ambiente 2. De igual forma, dentro de este ambiente se evidencia poca cantidad restos óseos humano, es posible establecer la presencia mínima de dos individuos, lo cual nos reafirma que se trata de un contexto funerario (Figura 12).

\section{Discusión}

Según lo registrado en los sitios arqueológicos Gentilmach'ay y Pushkanawasi, es posible definir que los pobladores prehispánicos de estas quebradas emplearon las partes altas de los cerros con el objetivo de utilizar las cavidades naturales para la construcción de estructuras funerarias o chullpas, las cuales estuvieron asociadas con las ceremonias fúnebres y con el culto a los ancestros (Duchesne y Chacama, 2012). Las chullpas son estructuras funerarias presentes desde el Intermedio Tardío (1000/1100-1450 d. C) y guardan relación directa con la evolución de los patrones funerarios Tiawanaku luego de su colapso, los cuales vieron mejoras técnicas y una mayor difusión en los Andes con la expansión del imperio Inca, registrándose este tipo de estructura desde Chachapoyas en el norte del Perú hasta el norte de Chile (Duchesne, 2005). El uso de chuIlpas es mencionado por diversos cronistas, como Pedro Cieza de León ([1553]1984), Bartolomé de las Casas ([1559] 1967) y Guaman Poma de Ayala ([1613] 2008); ellos confirmaron que el uso de estos espacios como sepultura y su rol dentro de la colectividad, por lo cual se considera que la construcción de estos espacios estaba destinada para los individuos de más alto prestigio de la comunidad (Kesseli y Parssinen, 2005). Sin embargo, estas estructuras también tendrían un rol social al ser utilizados como referentes geográficos o marca territorial para la población, con el objetivo de mantener el dominio social por parte de las elites aun después de la muerte (Tantaleán, 2006). Considerando lo registrado en ambas quebradas, es posible asociar por la técnica 
constructiva, que posiblemente se trate de dos contextos diferenciados por la importancia de los individuos sepultados dentro de ellas. En Gentilmach'ay, se observa un mejor acabado del material empleado, la construcción de una plataforma para habilitar el terreno para la elaboración de las chullpas y la presencia de otros ambientes asociados con las estructuras funerarias, las cuales se tratarían de espacios para colocar ofrendas durante la realización de las actividades conmemorativas. La organización espacial es diferente en Pushkanawasi, donde se observa el uso de material constructivo de menor calidad (rocas aristosas) y una técnica constructiva diferente, siendo ampliamente utilizado la concavidad del abrigo rocoso para la elaboración de un ambiente. Considerando que los posibles ejes económicos para esta zona, durante la época Inca, fueron el pastoreo (camélidos) y la textilería (como actividad derivada), posiblemente los dos sitios arqueológicos analizados tengan relación con dos grupos sociales diferenciados; esto se corroboraría con el tipo de ajuar funerario que presenten los individuos y su estado de salud.

La cerámica decorada registrada en superficie se encuentra asociada con el estilo Inca, siendo las líneas y una figura zoomorfa (ave) lo más representativo. Similar diseño se observa en las vasijas Inca (Horizonte Tardío) del Museo Larco, los cuales se encuentran asociadas a la costa sur del Perú. La fase a la cual pueda asociarse el material registrado es a la Inca Imperial, debido a que cuenta con sus principales características, como son la utilización de los colores blanco-crema, negro y rojo inglés; la representación de formas geométricas (triángulos), aves y "helechos", estando elaborados con un fino acabado y estilizados (Ravines, 2011; Villacorta, 2011) (Figura 17). La ocupación Inca es confirmada para la presente zona de estudio, corroborando la información brindada por los cronistas sobre la presencia inca en la provincia de Caylloma, sin embargo, el análisis de mayor material cerámico recuperado de estos contextos, producto de excavaciones arqueológicas, nos brindaría mayor información acerca de los estilos cerámicos presentes en las quebradas de Ucriamayo y Tulpaisa.

Figura 13: Fragmentos de cerámica con los diseños de "helechos" (A), línea zigzagueante (B), aves (C) característicos de la fase Inca Imperial. La figura D es un plato (MLo39830) con estilo Inca, proveniente de la costa sur (ML, 2010). La escala corresponde a $2 \mathrm{~cm}$.
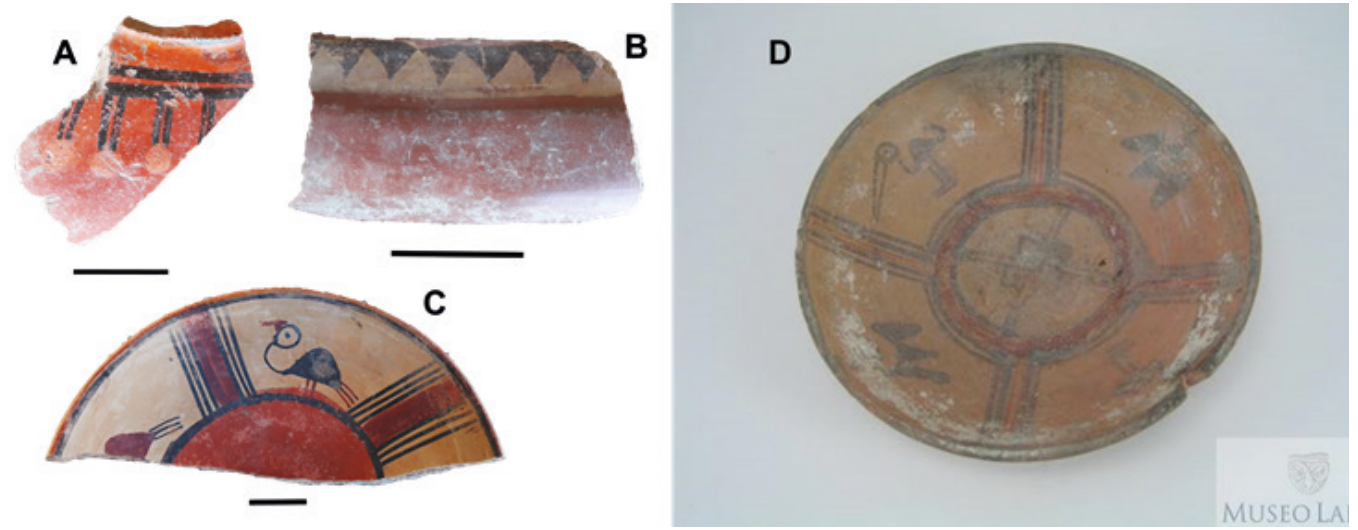

Los restos óseos expuestos en las chullpas se encuentra disturbados por el saqueo moderno, sin embargo, es posible observar múltiples individuos con deformación craneal dentro de los ambientes en Gentilmach'ay. La ubicación de ambas quebradas se encuentra en la zona alta del valle de Colca, históricamente considerado como territorio Collagua; este grupo tenía la costumbre de deformar el cráneo desde niños con la finalidad de elevar el cráneo para simular la forma del volcán Collaguata, ancestro tutelar de esta etnia (Pais, 2018). Posiblemente este 
sería el motivo de la deformación craneal registrada, ya para la época Inca, los collaguas (etnia de origen de la esposa secundaria del inca) tuvieron un rol importante y continuaron con sus costumbres a pesar de ser anexados. Igualmente, aunque no pueda definirse el ajuar de cada individuo, como los restos de textiles en Gentilmach'ay, una rueca de madera en Pushkanawasi y los fragmentos de cerámica con decoración, nos indicaría que se trata de personas con cierto rango dentro de la comunidad prehispánica establecida dentro de ambas quebradas.

No es posible aislar estas estructuras funerarias de la relación existente con los otros contextos arqueológicos presentes en las quebradas de Ucriamayo y Tulpaisa; a pesar que la investigación arqueológica es casi nula se identificó la posible área urbana donde se establecieron los habitantes de esta zona, la cual se encuentra denominado como el sitio arqueológico Tambomayo A, ubicado a $1.8 \mathrm{~km}$ y $4.9 \mathrm{~km}$ de los sitios de Gentilmach'ay y Pushkanawasi, respectivamente. En este asentamiento de grandes dimensiones es posible identificar múltiples estructuras circulares y cuadrangulares, siendo el área de mayor monumentalidad dentro de la quebrada de Ucriamayo.

La realización de excavaciones arqueológicas en estos dos sitios arqueológicos representaría una gran oportunidad para lograr conocer cuáles eran las características sociales y biológicas del poblador prehispánico en esta zona. Lograr correlacionar el tipo de estructura y las características de cada contexto funerario es fundamental para establecer diferencias sociales dentro de una misma comunidad, lo cual sería de gran apoyo para lograr confirmar qué tipo de economía practicaban los grupos asentados en estas quebradas. Con los restos óseos, es posible realizar múltiples investigaciones que nos brindaría información con relación a la salud del antiguo poblador (Favila Cisneros y Sarabia Gómez, 2010; Wester, 2010), características físicas (Verano, 1997, 1998), las alteraciones culturales a los cuales fueron sometidos en vida (Velasco, 20016; Torres-Rouff, 2019) y hasta una aproximación facial con el objetivo de conocer la posible fisionomía del poblador prehispánico (Guevara, 2014; Aparicio Resco y Azcune Fontecha, 2016). De igual forma, es posible estudiar los microrestos vegetales (granos de almidón y fitolitos) presentes en el sarro dental (Wesolowski, et al. 2007; Vásquez, et al. 2014), con la finalidad de identificar el tipo de vegetal consumido y lograr acercarnos a la reconstrucción de la dieta durante la época inca. Además, con el sedimento no disturbado presente en los diferentes ambientes de ambos sitios arqueológicos es posible estudiar el polen depositado de forma natural (Estévez y Pajón, 2006; González-Silvestre, et al. 2013) y reconstruir como era el entorno medioambiental en estas quebradas durante el Horizonte Tardío (1476 - 1532 d. C), con el objetivo de identificar posibles variaciones con el entorno natural actual.

\section{Conclusiones}

Los dos sitios arqueológicos registrados representan una importante oportunidad para lograr aproximarnos, no solo a las características sociales sino biológicas del poblador en las quebradas Ucriamayo y Tulpaisa, durante la ocupación Inca en el Horizonte Tardío. Gentilmach'ay cuenta con múltiples contextos funerarios con material cultural que puede ser asociada con el ajuar de los individuos presentes en sus ambientes 2 y 3 , asimismo, la organización espacial de las estructuras puede relacionarse con actividades de culto a los ancestros. Pushkanawasi, si bien no presenta la misma complejidad, puede considerarse como un área funeraria de menor rango si es comparado con el anterior sitio arqueológico mencionado. Lograr establecer la relación que existió entre ambos contextos arqueológicos dentro de la dinámica poblacional y la organización político-económica en la zona alta del valle del Colca daría nuevos alcances sobre la etnia Collagua durante la época Inca. 


\section{Agradecimiento}

Mi mayor gratitud a las áreas de gerencia y gestión ambiental de la U. M. Tambomayo (Compañía de Minas Buenaventura) por todas las facilidades brindadas al momento de registrar los sitios arqueológicos mencionados en la presente investigación. A Eduardo Eche Vega por la digitalización del material cerámico, a Carol Casafranca y Segundo Vásquez Sánchez por sus opiniones respecto a la investigación. A Daisi Gisel Gutierrez Mendoza por su apoyo en la redacción del escrito.

\section{Referencias bibliográficas}

Aparicio Resco, P.y Azcune Fontecha, I. (2016): Aproximaciones faciales en 3D para la socialización del patrimonio rural antropológico, La descommunal. Revista iberoamericana de patrimonio y comunidad 2: 529-543.

Asencios, A. (2015). Proyecto de Evaluación Arqueológica: Proyecto Minero Tambomayo- Sector I". Informe final. Unidad Minera Tambomayo. Armediam S.R.L. Buenaventura S.A.A. Arequipa.

Becerra, P.; Guevara, R.; Ramírez, K. y Rosas, G. (2005). Experiencias del trabajo de campo en el Valle del Colca - Arequipa. Revista de Antropología 3 (3): 334-353.

Castilla. Y. (2016). Proyecto de Evaluación Arqueológica: Proyecto Minero Tambomayo- Sector II. Informe final. Unidad Minera Tambomayo. Buenaventura S.A.A. Arequipa.

Cieza León, P. ([1553] 1984). La crónica del Perú. M. Ballesteros (editor). Madrid: Crónicas de América 4.

Doutriaux, M. (2002). Relaciones étnicas y económicas de poder: La conquista incaica en el valle del Colca, Arequipa. Boletín de arqueología PUCP 6: 411-432.

Duchesne, F. (2005). Tumbas de Coporaque. Aproximaciones a concepciones funerarias collaguas. Bulletin de l'Institut Français d'Études Andines 34 (3): 411-429.

Duchesne, F. y Chacama, J. (2012). Torres funerarias prehispánicas de los andes centro-sur: Muerte, ocupación del espacio y organización social. Estudio comparativo: Coporaque, Cañon del Colca (Perú), Chapiquiña, precordillera de Arica (Chile). Chungara 44 (4): 605619.

Estévez, Y. y Pajón, J. (2006). Consideraciones del análisis palinológico en reconstrucciones paleoambientales aplicadas a las investigaciones arqueológicas. En VIII Conferencia Internacional Antropologica 2006 "La antropología ante los nuevos retos de la humanidad". Ciudad de La Habana, Cuba, Noviembre 27-30 del 2006: 1-15.

Favila Cisneros, H. y Sarabia Gómez, E. (2010). La salud en la antigüedad prehispánica: un acercamiento bioantropológica de las condiciones de vida del Ecatepec antiguo. Ciencia ergo sum (17) 1: 37-45.

Fernández, J. (1980). La pintura Inka en cerámica. Obras Maestras del Arte en el antiguo Perú. Lima: Instituto Nacional de Cultura.

González-Silvestre, L.; Maldonado, A.; Nuñez, L.; Cartajena, I., Carrasco, C. y De Souza, P. (2013). Condiciones paleovegetacionales y asentamientos humanos durante el Formativo Temprano: Analisis de polen del sitio Tulán-85 (1.530/1.260-460/420 años CAL. A.C.), cuenca del Salar de Atacama. Chungara 45 (3): 378-410.

Guaman Poma de Ayala, F. ([1613] 2008). Nueva Corónica y Buen Gobierno. F. Pease (ed). México: Fondo de Cultura Económica.

Guevara, O. (2014). Reconstrucción arqueológica humana facial y sus funciones didácticas básicas para la nueva enseñanza-aprendizaje de la Historia peruana pre-hispánica. Convergencia Científica (1) 1:43- 61. 
Kesseli, R. y Pärssinen, M. (2005). Identidad étnica y muerte: torres funerarias (chullpas) como símbolos de poder étnico en el altiplano boliviano de Pakasa (1250-160o d. C.). Bulletin de I'Institut Français d'Études Andines 34 (3): 379-410

ML (Museo Larco) (2010). Catalogo en línea. Museo Larco. (16-11-19). https://www.museolarco. org/catalogo/ficha.php?id=41161

Las Casas, B. de ([1559] 1967) Apologética historia sumaria. E. O’Gorman (editor). Ciudad de México: Universidad Autónoma de México.

Pais, A. (2018). Por qué los collaguas y cabanas, dos antiguas civilizaciones de Perú, deformaban las cabezas de los bebés para que tuvieran forma de cono. BBC News Mundo. Publicado el 26 de febrero 2018. (15-11-2019). https://www.bbc.com/mundo/noticias-43198174

Ravines, R. (2011). Estilos de cerámica del antiguo Perú. Boletín de Lima 163 - 166:433-564.

Robles, R. (2008). Agricultura de riego y tradiciones en el valle del Colca. Revista de Antropología $6(6): 135-173$.

Tantaleán, H. (2006). Regresar para construir: prácticas funerarias e ideología(s) durante la ocupación Inka en Cutimbo, Puno-Perú. Chungara 38 (1): 129-143.

Torres-Rouff, C. (2019). Cranial modification and the shapes of heads acroos the Andes. En prensa, prueba corregida. International Journal of Paleopathology. DOI: https://doi.org/10.1016/j. ijpp.2019.06.007

Vásquez Sánchez, V.F.; Franco Jordán, R.; Rosales Tham, T. (2014). Almidones antiguos del cálculo dental de un entierro mochica de la Huaca Cao Viejo, complejo arqueológico El Brujo, costa norte del Perú. Revista Archaeobios (8) 1: 6- 16.

Velasco, M. (2016). Mortuary Tradition and Social Transformation during the Late Intermedia Period (A.D. 1100-1450) a Bioarchaeological analysis of above-ground burials in the Colca Valley, Perú. Tesis doctoral. Vanderbilt University, Nashville, Tennessee.

Verano, J. (1997). Human skeletal remains from Tomb 1, Sipán (Lambayeque river valley, Peru); and their social implications. Antiquity $71:$ 670-682.

Verano, J. (1998). Sacrificios humanos, desmembramientos y modificaciones culturales en restos osteológicos: Evidencias de las temporadas de investigación 1995-96 en Huaca de la Luna. En S. Uceda, E. Mujica y R. Morales (editores), Investigaciones en la Huaca de la Luna 1996 :159-171. Trujillo: Proyecto Arqueológico Huacas del Sol y de la Luna. Facultad de Ciencias Sociales y Universidad Nacional de La Libertad.

Villacorta, Y. (2011). Análisis de la cerámica Inca: Forma y diseños. Tesis de licenciatura. Universidad Nacional de San Antonio Abad del Cusco. Cusco.

Wesolowski, V.; De Souza, S.; Reinhard, K. y Ceccantini G. (2007). Grânulos de amido e fitólitos em cálculos dentários humanos: contribuição ao estudo do modo de vida e subsistência de grupos sambaquianos do litoral sul do Brasil. Revista do Museu de Arqueologia e Etnologia 17: 191- 210.

Wester, C. (2010). Chotuna-Chornancap. Templos, Rituales y Ancestros Lambayeque. Lambayeque: Unidad Ejecutora $\mathrm{N}^{\circ} 111$ "Naylamp Lambayeque". Museo Arqueológico Nacional BruningLambayeque. 